\title{
AUTOMORPHISM GROUPS OF HYPERELLIPTIC RIEMANN SURFACES
}

\author{
By E. Bujalance*, J. J. Etayo* and E. Martínez*
}

Hurwitz [3] proved that a Riemann surface of genus $g$ has at most $84(g-1)$ automorphisms. If the surface is hyperelliptic this bound may be very much shortened.

In this paper we obtain all surfaces of genus $g>3$ with more than $8(g-1)$ automorphisms, and their corresponding automorphism groups. As a consequence of these results, all hyperelliptic Riemann surfaces with more than $8(g-1)$ automorphisms appear to be symmetric.

The surfaces of low genus were studied by Wiman [12] and A. and I. Kuribayashi $[4,5]$.

The methods of our study of Riemann surfaces involve the representation of compact Riemann surfaces as quotient spaces of Fuchsian groups [6]. A Fuchsian group is a discrete subgroup of the group of orientable isometries of the hyperbolic plane $D$. If the quotient space $D / \Gamma, \Gamma$ being a Fuchsian group, is compact, then $\Gamma$ has the following presentation:

$$
\left\langle a_{1}, b_{1}, \cdots, a_{g}, b_{g}, x_{1}, \cdots, x_{r} \mid a_{1} b_{1} a_{1}^{-1} b_{1}^{-1} \cdots a_{g} b_{g} a_{g}^{-1} b_{g}^{-1} x_{1} \cdots x_{r}=x_{\imath}^{m_{\imath}}=1\right\rangle .
$$

Then we call $\left(g,\left[m_{1}, \cdots, m_{r}\right]\right)$ the signature of $\Gamma$ and $g$ is the genus of $D / \Gamma$. The numbers $m_{2}$ are called proper periods. When $X$ is a surface of genus $g$, it may be expressed as $D / \Gamma, \Gamma$ having signature $(g,[-])$.

If $G$ is a group of automorphisms of the Riemann surface $D / \Gamma$, then $G$ may be written as $\Gamma^{\prime} / \Gamma$, where $\Gamma^{\prime}$ is another Fuchsian group. A Fuchsian group $K$ with signature $\left(g,\left[m_{1}, \cdots, m_{r}\right]\right)$ has associated an area

$$
\text { Area }[K]=2 \pi\left(2 g-2+\sum_{\imath=1}^{r}\left(1-\frac{1}{m_{\imath}}\right)\right)=2 \pi|K|,
$$

and the order of $G=\Gamma^{\prime} / \Gamma$ is $|G|=|\Gamma| /\left|\Gamma^{\prime}\right|$.

Let now $D / \Gamma$ be a Riemann surface of genus $g$, and $G=\Gamma^{\prime} / \Gamma$ its group of automorphisms. If $|G|>8(g-1)$, then $\left|\Gamma^{\prime}\right|<\frac{2(g-1)}{8(g-1)}=\frac{1}{4}$. We list the Fuchsian groups $\Gamma^{\prime}$ with $\left|\Gamma^{\prime}\right|<\frac{1}{4}$.

* Partially supported by "Comisión Asesora de Investigación Científica y Técnica".

Received July 30, 1986. 

i) $(0,[2,3, m]) \quad m \geqq 7$
ii) $(0,[2,4, m]) \quad m \geqq 5$
iii) $(0,[2,5, m]) \quad 5 \leqq m \leqq 19$
iv) $(0,[2,6, m]) \quad 6 \leqq m \leqq 11$
v) $(0,[2,7, m]) \quad 7 \leqq m \leqq 9$
vi) $(0,[3,3, m]) \quad 4 \leqq m \leqq 11$
vii) $(0,[3,4,4])$
viii) $(0,[3,4,5])$
ix) $(0,[2,2,2,3])$.

A Riemann surface $X$ is hyperelliptic if it admits an involution $\phi$ such that $X / \phi$ has genus 0 . In terms of Fuchsian groups the surface $D / \Gamma$ is hyperelliptic if and only if there exists a unique Fuchsian group $\Gamma_{1}$ such that $\left|\Gamma_{1}: \Gamma\right|$ $=2$, and the signature of $\Gamma_{1}$ is $(0,[2, \cdots \cdots, 2])$ [9]. Furthermore, the element $\Gamma_{1} / \Gamma$ is central in the automorphism group of $D / \Gamma$, and so when $G=\Gamma^{\prime} / \Gamma$ is the automorphism group of the surface we have the following relations: $\Gamma \triangleleft \Gamma_{1} \triangleleft \Gamma^{\prime}$.

THEOREM. Let $X$ be a hyperelliptic Riemann surface of genus $g$ and $G$ its automorphism group. If $g>3$ and $|G|>8(g-1)$, then one of the following holds:

\begin{tabular}{clr}
\hline Genus of $X$ & \multicolumn{1}{c}{ Presentation of $G$} & Order of $G$ \\
\hline & $\left\langle a, b \mid a^{2}=b^{4}=(a b)^{m}=1, a b^{2}=b^{2} a\right\rangle$ & $4 m=8(g+1)$ \\
& $\left\langle a, b \mid a^{2}=b^{4}=(a b)^{m}=1, b^{2}=(a b)^{m / 2}\right\rangle$ & $2 m=8 g$ \\
5 & $\left\langle a, b \mid a^{2}=b^{3}=(a b)^{10}=1,(a b)^{5}=(b a)^{5}\right\rangle$ & 120 \\
9 & $\left\langle a, b \mid a^{2}=b^{5}=(a b)^{6}=1,(a b)^{3}=(b a)^{3}\right\rangle$ & 120 \\
6 & $\left\langle a, b \mid a^{2}=b^{6}=(a b)^{8}=1, b^{3}=(a b)^{4}\right\rangle$ & 48 \\
15 & $\left\langle a, b \mid a^{2}=b^{6}=(a b)^{10}=1, b^{3}=(a b)^{5}\right\rangle$ & 120 \\
5 & $\left\langle a, b \mid a^{3}=b^{4}=(a b)^{4}=1, a b^{2}=b^{2} a\right\rangle$ & 48 \\
14 & $\left\langle a, b \mid a^{3}=b^{4}=(a b)^{5}=1, a b^{2}=b^{2} a\right\rangle$ & 120 \\
\hline
\end{tabular}

Besides, in each case the surface is unique up to conformal equivalence.

Proof. Let $X=D / \Gamma$, with genus $g$. Since $X$ is hyperelliptic, there exists an automorphism $\phi$ of $X$, of order 2, and hence a Fuchsian group $\Gamma_{1}$ with signature $(0,[2, \cdots \cdots, 2])$ such that $\left|\Gamma_{1}: \Gamma\right|=2$. If $G=\Gamma^{\prime} / \Gamma$ is the automorphism group of $D / \Gamma$, then $\Gamma \triangleleft \Gamma_{1} \triangleleft \Gamma^{\prime}$. So there exists an epimorphism $\theta_{1}: \Gamma^{\prime} \rightarrow G_{1}=$ $G /\langle\phi\rangle$ whose kernel is isomorphic to $\Gamma_{1}$.

We look for the existence of this epimorphism $\theta_{1}$, according to the signature of $\Gamma^{\prime}$, i)-ix) above.

1) Let $\Gamma^{\prime}$ have signature i). Let $m$ be odd. We have $\theta_{1}: \Gamma^{\prime} \rightarrow G_{1}$ with 
kernel $\Gamma_{1}$. The order of $G_{1}$ is $\left|\Gamma_{1}\right| /\left|\Gamma^{\prime}\right|=\frac{g-1}{(m-6) / 6 m}=\frac{6 m(g-1)}{m-6}$. From [8, Cor. 2] since $m$ is odd, the proper periods of the kernel of $\theta_{1}$ are produced by the image of $x_{1}$. Hence $\theta_{1}\left(x_{1}\right)$ is 1 , and the number of proper periods in $\operatorname{ker} \theta_{1}$ is $\left|G_{1}\right|=\frac{6 m(g-1)}{m-6}$. We have $\frac{6 m(g-1)}{m-6}=2 g+2$, and $g=\frac{2 m-3}{m+3}$, impossible.

Now, if $m$ is even, the proper periods of $\operatorname{ker} \theta_{1}$ may be obtained from $x_{1}$ and $x_{3}$. Then its number is

$$
\alpha \frac{6 m(g-1)}{m-6}+\beta \frac{6 m(g-1)}{(m-6)(m / 2)},
$$

$\alpha$ (resp., $\beta$ ) being 1 when $\theta_{1}\left(x_{1}\right)$ (resp., $\theta_{1}\left(x_{3}\right)$ ) is 1 (resp., an element of order $m / 2$ ) and being 0 when $\theta_{1}\left(x_{1}\right)$ (resp., $\theta_{1}\left(x_{3}\right)$ ) is an element of order 2 (resp., order $m)[8$, Cor. 2]. So

$$
\alpha \frac{6 m(g-1)}{m-6}+\beta \frac{6 m(g-1)}{(m-6)(m / 2)}=2 g+2
$$

and this holds only for $\alpha=0, \beta=1, g=2$ or 5 . Thus the unique case in our scope is $\alpha=0, \beta=1, m=10, g=5$.

2) Let $\Gamma^{\prime}$ have signature ii). Now the order of $G_{1}$ is $\frac{4 m(g-1)}{m-4}$ and we distinguish again $m$ odd and $m$ even. By the same argument above, we consider the number of proper periods of $\operatorname{ker} \theta_{1}$ :

Let $m$ be odd. Then

$$
\alpha \frac{4 m(g-1)}{m-4}+\beta \frac{2 m(g-1)}{m-4}=2 g+2
$$

and in no case we obtain an integer value of $g$.

When $m$ is even.

$$
\alpha \frac{4 m(g-1)}{m-4}+\beta \frac{2 m(g-1)}{m-4}+\gamma \frac{8(g-1)}{m-4}=2 g+2 .
$$

The possibilities that appear now are:

$$
\begin{aligned}
& \text { (*) } \alpha=0, \quad \beta=1, \quad \gamma=0, \quad g=\frac{m-2}{2} \text {, } \\
& \alpha=1, \quad \beta=0, \quad \gamma=0, \quad m=12, \quad g=2, \\
& \alpha=0, \quad \beta=0, \quad \gamma=1, \quad m=6, \quad g=3, \\
& \text { (*) } \quad \alpha=0, \quad \beta=1, \quad \gamma=1, \quad g=\frac{m}{4} \text {. }
\end{aligned}
$$

For our purposes we will deal only with the starred cases. 
3) Let $\Gamma^{\prime}$ have signature iii). The order of $G_{1}$ is $\frac{10 m(g-1)}{3 m-10}$.

If $m$ is odd,

$$
\frac{10 m(g-1)}{3 m-10}=2 g+2, \quad \text { impossible. }
$$

If $m$ is even,

$$
\alpha \frac{10 m(g-1)}{3 m-10}+\beta \frac{20(g-1)}{3 m-10}=2 g+2
$$

and it holds just for $\alpha=0, \beta=1, m=6, g=9$.

4) Let $\Gamma^{\prime}$ have signature iv). Then the order of $G_{1}$ is $\frac{3 m(g-1)}{m-3}$ and the number of proper periods of $\operatorname{ker} \theta_{1}$ is, when $m$ is odd,

$$
\alpha \frac{3 m(g-1)}{m-3}+\beta \frac{m(g-1)}{m-3}=2 g+2
$$

and then $\alpha=1, \beta=1, m=9, g=2$, what is out of our scope.

If $m$ is even,

$$
\alpha \frac{3 m(g-1)}{m-3}+\beta \frac{m(g-1)}{m-3}+\gamma \frac{6(g-1)}{m-3}=2 g+2
$$

and then we have

$$
\begin{aligned}
& \alpha=1, \quad \beta=0, \quad \gamma=1, \quad m=8, \quad g=2, \\
& \alpha=0, \quad \beta=1, \quad \gamma=1, \quad m=6, \quad g=3, \\
& \text { (*) } \alpha=0, \quad \beta=1, \quad \gamma=1, \quad m=8, \quad g=6 \text {, } \\
& \text { (*) } \alpha=0, \quad \beta=1, \quad \gamma=1, \quad m=10, \quad g=15 \text {, } \\
& \alpha=1, \quad \beta=1, \quad \gamma=1, \quad m=12, \quad g=2,
\end{aligned}
$$

from which the starred cases are the interesting ones.

5) Let $\Gamma^{\prime}$ have signature v). The order of $G_{1}$ is $\frac{14 m(g-1)}{5 m-14}$. If $m=7$ or 9 we have

$$
\frac{14 m(g-1)}{5 m-14}=2 g+2, \quad \text { impossible. }
$$

If $m=8$,

$$
\alpha \frac{112(g-1)}{26}+\beta \frac{28(g-1)}{26}=2 g+2, \quad \text { impossible. }
$$

6) When $\Gamma^{\prime}$ has signature vi), the order of $G_{1}$ is $\frac{3 m(g-1)}{m-3}$. The kernel of 
$\theta_{1}$ has proper periods only when $m$ is even, and their number is

$$
\frac{6 m(g-1)}{m-3}=2 g+2
$$

holding only when $m=4, g=2$, out of our hypothesis.

7) Let $\Gamma^{\prime}$ have signature vii). The order of $G_{1}$ is $6(g-1)$ and the number of proper periods of $\operatorname{ker} \theta_{1}$ is $\alpha 3(g-1)$, with $\alpha=1$ or 2 ; if $\alpha=1, g=5$, and if $\alpha=2, g=2$.

8) If $\Gamma^{\prime}$ has signature viii), the order of $G_{1}$ is $\frac{60(g-1)}{13}$ and the number of proper periods is

$$
\frac{30(g-1)}{13}=2 g+2
$$

holding for $g=14$.

9) If $\Gamma^{\prime}$ has signature ix), the order of $G_{1}$ is $6(g-1)$ and there are $\alpha 6(g-1)$ proper periods, $1 \leqq \alpha \leqq 3$. When $\alpha=1$, it is $g=2$ and the other cases are impossible.

Now we check that all these cases actually hold. Since $G_{1}$ is a group of automorphisms of $X /\langle\phi\rangle$, a surface of genus 0 , by $[1, \S \S 4.3,4.4]$, this group must be one of the following: $C_{q}, D_{q}, A_{4}, S_{4}$ or $A_{5}$. (In what follows we use for finite groups the notation of [1]). In each of the cases we construct the epimorphism $\theta$ satisfying $\pi \theta=\theta_{1}$, where $\pi: G \rightarrow G_{1}=G /\langle\phi\rangle$ is the canonical projection. Since $\operatorname{ker} \theta$ has no proper periods, $\theta\left(x_{\imath}\right)$ must have order $m_{\imath}$. We study separately the eight possibilities.

I. $\Gamma^{\prime}:(0,[2,3,10]), g=5,\left|G_{1}\right|=60, \alpha=0, \beta=1$. Then the signature of $\Gamma_{1}$ is $\left(0,\left[2^{(12)}\right]\right.$ ) (where $2^{(p)}$ means that there exist $p$ proper periods equal to two), and $\theta_{1}: \Gamma^{\prime} \rightarrow G_{1}$ is defined [9] by

$$
\theta_{1}\left(x_{1}\right)=x, \quad \theta_{1}\left(x_{2}\right)=y, \quad \theta_{1}\left(x_{3}\right)=(x y)^{-1} .
$$

satisfying $x^{2}=y^{3}=(x y)^{5}=1$. Hence $G_{1}=A_{5}$. Now the epimorphism $\theta: \Gamma^{\prime} \rightarrow G$ with kernel $\Gamma$ is given by

$$
\theta\left(x_{1}\right)=a, \quad \theta\left(x_{2}\right)=b, \quad \theta\left(x_{3}\right)=(a b)^{-1},
$$

with $a^{2}=b^{3}=(a b)^{10}=1$. Since $\pi \theta=\theta_{1},(a b)^{5}$ is a central element and so $(a b)^{5}=$ $(b a)^{5}$. This group is just the group

$$
\left\langle R, S \mid R^{10}=S^{3}=(R S)^{2}=\left(R^{-4} S\right)^{2}=1\right\rangle
$$

described in [11, p. 46]. For, call $a=R S, b=S$. Then $S^{3}=b^{3}=1 ;(R S)^{2}=a^{2}=1$; since $a b=S^{-1} R^{-1} S$, the order of $R$ is the one of $a b$, i.e., 10 ; finally $\left(R^{-4} S\right)^{2}=$ $\left((b a)^{4} b\right)^{2}=(a b)^{10}=1$. By the definition of the epimorphism, $D /$ ker $\theta$ is a Riemann surface of genus $g$, and its hyperellipticity comes from the automorphism 
$(a b)^{5}$.

II. $\Gamma^{\prime}:(0,[2,4, m]), m$ even, $g=(m-2) / 2,\left|G_{1}\right|=4(g+1)=2 m, \alpha=0, \beta=1$, $\gamma=0$. The signature of $\Gamma_{1}$ is $\left(0,\left[2^{(2 g+2)}\right]\right)$ and $\theta_{1}: \Gamma^{\prime} \rightarrow G_{1}$ is defined by

$$
\theta_{1}\left(x_{1}\right)=x, \quad \theta_{1}\left(x_{2}\right)=y, \quad \theta_{1}\left(x_{3}\right)=(x y)^{-1},
$$

$x^{2}=y^{2}=(x y)^{m}=1$, and obviously $G_{1}=D_{m}$. The epimorphism $\theta: \Gamma^{\prime} \rightarrow G$ with kernel $\Gamma$ is given by

$$
\theta\left(x_{1}\right)=a, \quad \theta\left(x_{2}\right)=b, \quad \theta\left(x^{3}\right)=(a b)^{-1},
$$

with $a^{2}=b^{4}=(a b)^{m}=1$. Since $\pi \theta=\theta_{1}, b^{2}$ is a central element and so $a b^{2}=b^{2} a$. This group has order $8(g+1)$ and has presentation

$$
\left\langle T, U \mid T^{4}=U^{2(g+1)}=(T U)^{2}=\left(T^{-1} U\right)^{2}=1\right\rangle
$$

(see [7, Th. 4]). Effectively, we call $b=T, a=T U$, and the presentation follows at once. Now $D / \operatorname{ker} \theta$ is a Riemann surface of genus $g$ and $b^{2}$ gives the hyperellipticity.

III. $\Gamma^{\prime}:(0,[2,4, m]), m$ a multiple of $4, g=m / 4,\left|G_{1}\right|=4 g=m, \alpha=0, \beta=1$, $\gamma=1 . \quad \Gamma_{1}$ is again $\left(0,\left[2^{(2 g+2)}\right]\right)$ and $\theta_{1}$ is given by

$$
\theta_{1}\left(x_{1}\right)=x, \quad \theta_{1}\left(x_{2}\right)=y, \quad \theta_{1}\left(x_{3}\right)=(x y)^{-1}
$$

satisfying $x^{2}=y^{2}=(x y)^{m / 2}=1$, and $G_{1}$ is $D_{m / 2}$. Now $\theta: \Gamma^{\prime} \rightarrow G$ is defined by

$$
\theta\left(x_{1}\right)=a, \quad \theta\left(x_{2}\right)=b, \quad \theta\left(x_{3}\right)=(a b)^{-1},
$$

$a^{2}=b^{4}=(a b)^{m}=1$, and by unicity of the central element, $b^{2}=(a b)^{m / 2}$. Consider the group $C_{m}=\left\langle y \mid y^{m}=1\right\rangle, C_{2}=\left\langle x \mid x^{2}=1\right\rangle$, and $C_{m} \times_{\phi} C_{2}$ with $\phi: C_{2} \rightarrow$ Aut $\left(C_{m}\right)$, $\phi(1)=I d, \phi(x)=\psi, \phi(y)=y^{(m / 2)-1}$. In this situation $a=(x, 1), a b=(1, y)$ and $G$ is so $C_{m} \rtimes C_{2}$. D $D / \operatorname{ker} \theta$ is a Riemann surface of genus $g$ and $b^{2}=(a b)^{m / 2}$ gives the hyperellipticity.

IV. $\Gamma^{\prime}:(0,[2,5,6]), g=9,\left|G_{1}\right|=60, \alpha=0, \beta=1$. The signature of $\Gamma_{1}$ is $\left(0,\left[2^{(20)}\right]\right)$ and $\theta_{1}$ is defined by

$$
\theta_{1}\left(x_{1}\right)=x, \quad \theta_{1}\left(x_{2}\right)=y, \quad \theta_{1}\left(x_{3}\right)=(x y)^{-1} .
$$

satisfying $x^{2}=y^{5}=(x y)^{3}=1, G_{1}$ being so $A_{5}$. Now $\theta$ is given by

$$
\theta\left(x_{1}\right)=a, \quad \theta\left(x_{2}\right)=b, \quad \theta\left(x_{3}\right)=(a b)^{-1},
$$

with $a^{2}=b^{5}=(a b)^{6}=1$, and $(a b)^{3}=(b a)^{3}$. By $[1, \mathrm{f} .76] G$ is the quotient of the group $2[6] 5$ by adding the relation "the central element has order two". $X$ is a hyperelliptic surface and $\phi=(a b)^{3}$.

V. $\Gamma^{\prime}:(0,[2,6,8]), g=6,\left|G_{1}\right|=24, \alpha=0, \beta=1, \gamma=1$. The signature of $\Gamma_{1}$ is $\left(0,\left[2^{(14)}\right]\right)$ and $\theta_{1}$ is defined by

$$
\theta_{1}\left(x_{1}\right)=x, \quad \theta_{1}\left(x_{2}\right)=y, \quad \theta_{1}\left(x_{3}\right)=(x y)^{-1} .
$$


with $x^{2}=y^{3}=(x y)^{4}=1$. So $G_{1}$ is $S_{4}$. Now the epimorphism $\theta$ is given by

$$
\theta\left(x_{1}\right)=a, \quad \theta\left(x_{2}\right)=b, \quad \theta\left(x_{3}\right)=(a b)^{-1} .
$$

satisfying $a^{2}=b^{6}=(a b)^{8}=1$, and, as above, $b^{3}=(a b)^{4} . \quad G$ is the group $\langle 3,4 \mid 2 ; 2\rangle$ $\cong S_{4} \times C_{2}[1$, p. 72$], X$ is a hyperelliptic surface and $\phi=b^{3}=(a b)^{4}$.

VI. $\Gamma^{\prime}:(0,[2,6,10]), g=15,\left|G_{1}\right|=60, \alpha=0, \beta=1, \gamma=1 . \quad \Gamma_{1}$ has signature $\left(0,\left[2^{(32)}\right]\right)$ and the epimorphism $\theta_{1}$ is defined by

$$
\theta_{1}\left(x_{1}\right)=x, \quad \theta_{1}\left(x_{2}\right)=y, \quad \theta_{1}\left(x_{3}\right)=(x y)^{-1},
$$

with $x^{2}=y^{3}=(x y)^{5}=1$, and $G_{1}$ is $A_{5}$. The epimorphism $\theta$ is given by

$$
\theta\left(x_{1}\right)=a, \quad \theta\left(x_{2}\right)=b, \quad \theta\left(x_{3}\right)=(a b)^{-1}
$$

with $a^{2}=b^{6}=(a b)^{10}=1$ and $b^{3}=(a b)^{5} . \quad G$ is the group $\langle 3.5 \mid 2 ; 2\rangle \cong A_{5} \times C_{2}[1$, p. 72]. $X$ is a hyperelliptic surface and $\phi=b^{3}=(a b)^{5}$.

VII. $\Gamma^{\prime}:(0,[3,4,4]), g=5,\left|G_{1}\right|=24, \alpha=1$. In this case the signature of $\Gamma_{1}$ is $\left(0,\left[2^{(12)}\right]\right)$. The epimorphism $\theta_{1}$ is defined by

$$
\theta_{1}\left(x_{1}\right)=x, \quad \theta_{1}\left(x_{2}\right)=y, \quad \theta_{1}\left(x_{3}\right)=(x y)^{-1},
$$

with $x^{3}=y^{2}=(x y)^{4}=1$, and $G_{1}$ is $S_{4}$. The epimorphism $\theta$ is given by

$$
\theta\left(x_{1}\right)=a, \quad \theta\left(x_{2}\right)=b, \quad \theta\left(x_{3}\right)=(a b)^{-1},
$$

with $a^{3}=b^{4}=(a b)^{4}=1$, and $a b^{2}=b^{2} a . \quad G$ is the quotient of the group $3[4] 4$ ( $[1$, p. 76$])$ by adding the relation "the central element has order two". $X$ is a hyperelliptic surface and $\phi=b^{2}$.

VIII. $\Gamma^{\prime}:(0,[3,4,5]), g=14,\left|G_{1}\right|=60 . \Gamma_{1}$ has signature $\left(0,\left[2^{(30)}\right]\right)$ and $\theta_{1}$ is given by

$$
\theta_{1}\left(x_{1}\right)=x, \quad \theta_{1}\left(x_{2}\right)=y, \quad \theta_{1}\left(x_{3}\right)=(x y)^{-1},
$$

satisfying $x^{3}=y^{2}=(x y)^{5}=1$. We have $G_{1}=A_{5}$ and $\theta$ is defined by

$$
\theta\left(x_{1}\right)=a, \quad \theta\left(x_{2}\right)=b, \quad \theta\left(x_{3}\right)=(a b)^{-1},
$$

with $a^{3}=b^{4}=(a b)^{5}=1$ and $a b^{2}=b^{2} a . \quad G$ is the quotient of the group $3[4] 5$ ( $[1$, p. 76$])$ by adding the relation "the central element has order two". $X$ is a hyperelliptic surface and $\phi=b^{2}$.

Observe that in all cases I-VIII $\Gamma^{\prime}$ is a triangle group and so its Teichmüller space has dimension 0 . Besides, the epimorphism $\theta$ is unique modulo Aut $\left(\Gamma^{\prime}\right)$ and Aut $(G)$. Hence, using $[2, \S 3]$, the surface $D / \operatorname{ker} \theta$ is unique in each case, up to conformal equivalence.

Corollary. If $X$ is a hyperelliptic Riemann surface of genus $g>3$ with $\mid$ Aut $(X) \mid>8(g-1)$, then $X$ is symmetric. 
Proof.

Cases I and III come from [10, note in p. 24 and th. 4, resp.], and case II from $\left[11\right.$, p. 46]. In cases IV-VIII, since $a \rightarrow a^{-1}, b \rightarrow b^{-1}$ is an automorphism of the group, it follows from $[10$, th. 2] that $X$ is symmetric.

The authors wish to thank the referee for his helpful suggestions.

\section{REFERENCES}

[1] Coxeter, H.S.M., And Moser, W.O. J., "Generators and relations for discrete groups". Ergeb. der Math., 14. Springer. Berlin, etc. 1980 (4th ed.).

[2] Harvey, W. J., "On branch loc1 in Teichmüller space". Trans. Amer. Math. Soc. 153 (1971), 387-399.

[3] Hurwitz, A., "Über algebraische Gebilde mit eindeutigen Transformationen in sich". Math. Ann. 41 (1893), 403-442.

[4] KuRibayashi, A., "On analytic families of compact Riemann surfaces with nontrivial automorphisms". Nagoya Math. J. 28 (1966), 119-165.

[5] Kuribayashi, I., "Hyperelliptic AM curves of genus three and associated representations". Preprint.

[6] Macbeath, A. M., "Discontınuous groups and birational transformations". Proc. Dundee Summer School 1961, 59-75.

[7] Maclachlan, C., "A bound for the number of automorphisms of a compact Riemann surface". J. London Math. Soc. 44 (1969), 265-272.

[8] Maclachlan, C., Maximal normal Fuchsian groups. IIl. J. Math. 15 (1971), 104113.

[9] Maclachlan, C., Smooth coverings of hyperelliptic surfaces. Quart. J. Math. Oxford. (2) 22 (1971), 117-123.

[10] Singerman, D., Symmetries of Riemann surfaces with large automorphism group. Math. Ann. 210 (1974), 17-32.

[11] Singerman, D., Symmetries and pseudo-symmetries of hyperelliptic "isurfaces. Glasgow Math. J. 21 (1980), 39-49.

[12] Wiman, A., Über die hyperelliptischen Curven und diejenigen vom _Geschlechte $p=3$, welche eindeutigen Transformationen in sich zulassen, Bihang Kongl. Svenska Vetenskapsakademiens Handlingar, Stockholm 1895-96.

E. Bujalance and E. Martínez

Departmento de Matemática Fundamental

FACUlTAd DE Ciencias

U. N.E.D.

28040 MADRID-SPAIN

J. J. ETAYo

Departmento de Geometria y Topologia

Facultad de C. Matemáticas

UnIVERSIDAD COMPLUTENSE

28040 MADRID-SPAIN 